PHYSICAL REVIEW A 92, 033411 (2015)

$\mathcal{S}^{\circ}$

\title{
Using laser-induced Coulomb explosion of aligned chiral molecules to determine their absolute configuration
}

\author{
Lauge Christensen and Jens H. Nielsen* \\ Department of Physics and Astronomy, Aarhus University, Ny Munkegade 120, DK-8000 Aarhus C, Denmark \\ Craig S. Slater, Alexandra Lauer, and Mark Brouard \\ Physical and Theoretical Chemistry Laboratory, Department of Chemistry, University of Oxford, South Parks Road, \\ Oxford OX1 3QZ, United Kingdom \\ Henrik Stapelfeldt \\ Department of Chemistry, Aarhus University, Langelandsgade 140, DK-8000 Aarhus C, Denmark
}

(Received 8 April 2015; published 9 September 2015)

\begin{abstract}
The absolute configuration of chiral molecules in the gas phase is determined by femtosecond laser-induced Coulomb explosion and recording of the emission direction of critical ionic fragments. The spatial orientation of the 3,5-dibromo-3',5'-diflouro-4'-cyanobiphenyl molecules studied is determined by a combination of laserinduced alignment and time-of-flight measurement of $\mathrm{F}^{+}$ion recoils. Hereby, the enatiomeric state of the molecules could be uniquely identified through analysis of the correlation of the angular distribution of $\mathrm{F}^{+}$and $\mathrm{Br}^{+}$ions. The method will apply to a range of molecules and allow imaging of chiral changes on ultrafast time scales.
\end{abstract}

DOI: 10.1103/PhysRevA.92.033411

PACS number(s): $33.80 . \mathrm{Rv}, 33.15 . \mathrm{Hp}, 42.50 . \mathrm{Hz}$

\section{INTRODUCTION}

Molecules that exist in two nonsuperimposable mirror images are termed chiral and the two mirror images are called enantiomers. Since the two enantiomers often have different chemical properties, the ability to identify the specific handedness of a chiral molecule, often referred to as the absolute configuration (AC), is of central importance. This is, however, a nontrivial task because the two enantiomers have identical physical properties.

The most widely used technique for retrieving the AC, applying to molecules in crystalline form, is anomalous X-ray diffraction [1], whereas for molecules in solution vibrational circular dichroism [2] or vibrational optical activity [3] compared to calculated spectra can provide the AC. Recently, the determination of the AC of gas phase molecules has been the subject of considerable attention and addressed by at least three methods. In the first, photoelectron circular dichroism, the difference in angle-resolved photoelectron emission from pure enantiomers obtained with left-handed and right-handed circularly polarized light is measured. Upon comparison with theoretical simulations, the AC can be assigned for ionization induced both by synchrotron light $[4,5]$ and by strong laser pulses [6,7]. The second, microwave spectroscopy based on broadband pulsed radiation, in comparison with simulations, makes it possible to determine the AC of molecules that possess components of the electrical dipole moment along all three principal molecular axes [8,9]. In the third molecules are multiply ionized by beams of highly charged ions [10], by collisions with thin metal foils [11], or by intense laser pulses [12], causing Coulomb explosion of

\footnotetext{
*Present address: Research Software Development Group, Research IT Services, University College London, Gower Street, London WC1E 6BT, United Kingdom.
}

the molecules. The emission directions and kinetic energies of the recoiling fragments are measured and provide direct information about the nuclear structure including the AC without any need for simulations or modeling.

The Coulomb explosion imaging studies on the AC have hitherto employed detection of the three-dimensional (3D) momentum vectors of all (or most of the) fragments from each individual molecule that undergoes Coulomb explosion. Using subsequent coincidence analysis, very extensive structural information is accessible [12], but there are also limitations. First, the data acquisition time is high, which may be prohibitive for future time-resolved studies where many successive measurements of the molecular structure are required. Second, it is not obvious if the technique is usable for molecules containing more than five to ten atoms where a large number of different fragments may be produced.

In the current work we introduce an alternative approach to the determination of the AC through laser-induced Coulomb explosion imaging that overcomes these two limitations. It relies on first identifying the spatial orientation of the molecules by laser aligning them prior to the Coulomb explosion process and detecting the recoil direction of one fragment ion species through time-of-flight measurements. Hereafter, the detection of the correlations of the angular distributions of two critical fragment species, implemented by a covariance analysis of $2 \mathrm{D}$ ion images, yields the AC. Our method is demonstrated for 3,5-dibromo-3', $5^{\prime}$-diflouro-4'-cyanobiphenyl (BFCbP), an axially chiral molecule consisting of 23 atoms $\left(\mathrm{C}_{13} \mathrm{H}_{5} \mathrm{Br}_{2} \mathrm{~F}_{2} \mathrm{~N}\right)$ and as such representing a significantly more complex system than those studied so far.

\section{PRINCIPLE OF THE METHOD}

The BFCbP molecule, schematically shown in Fig. 1, possesses an axis of chirality (along the $\mathrm{C}-\mathrm{C}$ bond joining 
(d)
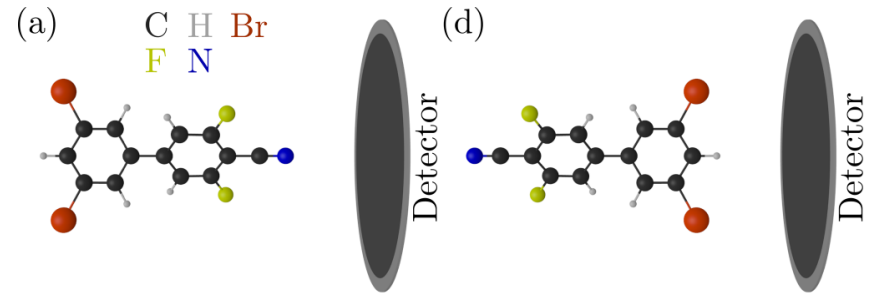

(b)

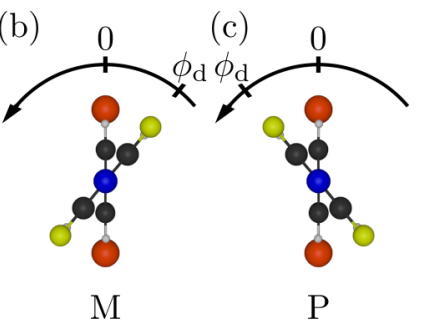

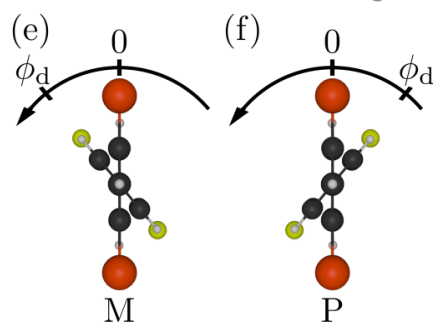

FIG. 1. (Color online) Effect of orientation and chirality on the sign of the dihedral angle as seen from the detector. (a) Orientation that leads to an early $\mathrm{F}^{+}$ion signal. The sign of the dihedral angle seen by the detector is shown for the (b) $M$ and (c) $P$ enantiomers. (d) Orientation that leads to a late $\mathrm{F}^{+}$ion signal. The sign of the dihedral angle seen by the detector is shown for the (e) $M$ and (f) $P$ enantiomers. The circular axes in (b), (c), (e), and (f) indicate $\phi_{\mathrm{d}}$. The atoms in the molecule are labeled by color.

the two benzene rings) [13] rather than a chiral center. The two enantiomers are labeled by $P$ (plus), referring to the form of a right-handed helix around the axis of chirality, and by $M$ (minus), referring to the form of a left-handed helix. Both enantiomers are shown in Fig. 1. The experiment employs laser-induced $1 \mathrm{D}$ alignment of the molecules to fix the most polarizable axis (the chiral $\mathrm{C}-\mathrm{C}$ axis) along the direction perpendicular to the detector [Figs. 1(a) and 1(d)]. When viewed from the detector the molecules therefore appear as one of four structures, determined by the enantiomeric state and by the direction in which the $\mathrm{CN}$ group points, illustrated in Figs. 1(b), 1(c), 1(e), and 1(f). This end-view geometry provides a direct view of the dihedral angle $\phi_{\mathrm{d}}$, the angle from the Br-containing to the F-containing benzene ring, and it has previously been used to determine the dihedral angle for BFCbP [14] and a similar molecule [15].

Figures 1(b) and 1(c) show that, seen from the detector, $\phi_{\mathrm{d}}=-39^{\circ}\left(=321^{\circ}\right)$ for the $M$ enantiomer and $\phi_{\mathrm{d}}=39^{\circ}$ for the $P$ enantiomer when the $\mathrm{CN}$ group points toward the detector (the equilibrium value of $\phi_{\mathrm{d}}$ is $39^{\circ}$ according to density-functional theory [14]). If instead the $\mathrm{CN}$ group is turned away from the detector, the $M$ enantiomer yields $\phi_{\mathrm{d}}=39^{\circ}$ and the $P$ enantiomer $\phi_{\mathrm{d}}=-39^{\circ}$ [see Figs. 1(e) and $1(\mathrm{f})]$. Thus, the sign of the dihedral angle uniquely identifies the enantiomer, but only if either all molecules in a sample have their $\mathrm{CN}$ group pointing in the same direction prior to the measurement or if some observable recorded in the experiment allows the identification of those molecules that have their $\mathrm{CN}$ group pointing in the same direction. The former criteria would correspond to a sample of perfectly oriented molecules. Although it has been demonstrated that the orientation, induced by the combined effect of the alignment laser field and the static electric field in the spectrometer used for the experiment, can be very strong for several molecular species $[16,17]$, we found that this is not the case for BFCbP. Instead we show that by recording the time it takes the recoiling ions from the Coulomb explosion to reach the detector, in addition to the $2 \mathrm{D}$ ion images that determine $\phi_{\mathrm{d}}$, it is possible to identify and thus analyze the subset of molecules that have their $\mathrm{CN}$ group pointing either toward or away from the detector.

\section{EXPERIMENTAL SETUP}

The experimental setup was described before [18] and only a brief account is included here. A cold molecular beam of BFCbP seeded in helium was crossed at $90^{\circ}$ by two laser beams. The first beam, consisting of linearly polarized pulses from a Nd:YAG laser (YAG pulse, $\lambda=1064 \mathrm{~nm}, \tau_{\mathrm{FWHM}}=$ $10 \mathrm{~ns}, E_{\text {pulse }}=150 \mathrm{~mJ}$, and $I_{0}=8.0 \times 10^{11} \mathrm{~W} \mathrm{~cm}^{-2}$ ), was used to adiabatically align the molecules in one dimension. The second beam, consisting of intense, circularly polarized pulses (probe pulse, $800 \mathrm{~nm}, 30 \mathrm{fs}, 103 \mu \mathrm{J}$, and $3.4 \times$ $10^{14} \mathrm{~W} \mathrm{~cm}^{-2}$ ), was used to measure the dihedral angle of the aligned molecules by Coulomb exploding the molecules and subsequently recording the emission direction of recoiling $\mathrm{F}^{+}$and $\mathrm{Br}^{+}$fragments. In practice, all cationic ions created by the probe pulse were accelerated by a three-electrode ion optics assembly, optimized to effect velocity-map ion imaging [19], and focused onto a detector consisting of a pair of microchannel plates and a P47 phosphor screen. The phosphor screen was backed by a pixel imaging mass spectrometry (PImMS) camera [20-22].

For each ion event imaged on the phosphor screen the PImMS camera recorded the position, i.e., the velocity vector in the detector plane, and placed a time stamp with a 12.5-ns resolution on the event. From the time stamping the time-offlight (TOF) spectrum is obtained and the result is displayed in Fig. 2. It shows that a number of ionic species is created, including $\mathrm{F}^{+}$ions and $\mathrm{Br}^{+}$ions, which are the essential observables in the current work. The resolution of the time stamping is sufficient that 2D images of each individual ion species appearing in the TOF spectrum are obtained and we exploit this to determine the dihedral angle through covariance analysis of the $\mathrm{F}^{+}$and $\mathrm{Br}^{+}$ion images $[14,15]$. In addition,

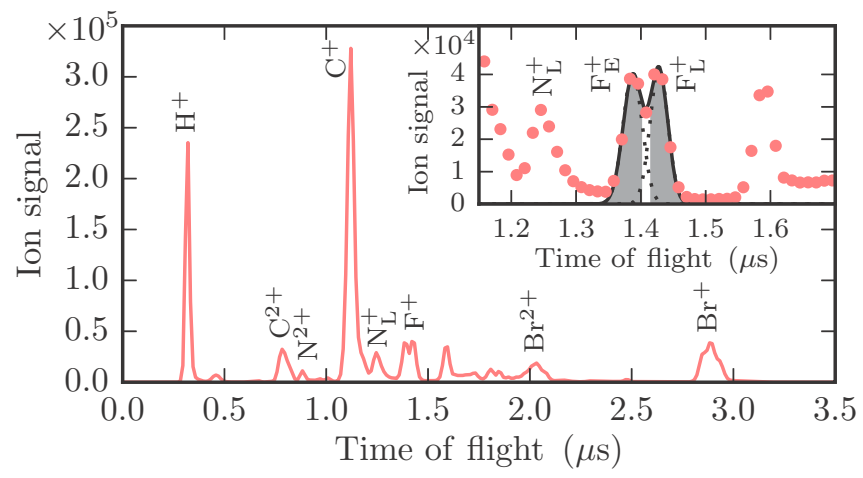

FIG. 2. (Color online) Time-of-flight mass spectrum recorded for $1 \mathrm{D}$ aligned BFCbP molecules ionized by the probe pulse. The inset shows a magnification of the region around the arrival time of the $\mathrm{F}^{+}$ions with the experimental data marked by light red circles. The $E$ and $L$ subscripts label the early and late peaks used to identify the orientation of the molecules (see the text for details). 
the resolution is also sufficient to identify the orientation of the parent molecule to each $\mathrm{F}^{+}$ion event as we discuss in the following section.

\section{EXPERIMENTAL RESULTS AND DATA ANALYSIS}

For molecules oriented as in Fig. 1(a), the $\mathrm{F}^{+}$and $\mathrm{N}^{+}$ions $\left(\mathrm{Br}^{+}\right.$ions) are ejected in the direction toward (away from) the detector and will therefore arrive earlier (later) at the detector than ions without kinetic energy. For molecules with the opposite orientation [Fig. 1(d)] the $\mathrm{F}^{+}$and $\mathrm{N}^{+}$ions $\left(\mathrm{Br}^{+}\right.$ ions) will instead arrive late (early). As a result, the peaks in the TOF spectrum corresponding to ions of the $\mathrm{N}, \mathrm{Br}$, and $\mathrm{F}$ atoms are expected to split into an early and a late peak. Figure 2 shows that for the singly and doubly charged nitrogen ions the late peaks are well resolved, whereas the early peaks cannot be identified because they overlap with the much stronger carbon ion peaks. For the $\mathrm{F}^{+}$ions both the early and the late peaks are observed (most easily seen in the inset on Fig. 2) even though the $\mathrm{F}^{+}$ions do not recoil with the full velocity vector along the TOF direction. The $\mathrm{Br}^{+}$signal should in principle also exhibit a splitting, but the almost equal abundance of the two bromine isotopes creates four subpeaks that overlap in a way to wash out the splitting.

The important consequence is that the splitting of the $\mathrm{F}^{+}$signal allows the determination of the parent molecule orientation for each $\mathrm{F}^{+}$event detected with the PImMS camera simply by determining if the time stamp of the $\mathrm{F}^{+}$ion falls within the region defining the early peak [orientation as in Fig. 1(a)] or within the region defining the late peak [orientation as in Fig. 1(d)]. Thus, if the data analysis is restricted to the $\mathrm{F}^{+}$events with a time stamp in the early peak or in the late peak, then the two enantiomers will be uniquely identified by $\phi_{\mathrm{d}}$. The time ranges of the early peak $(1.34-1.40 \mu \mathrm{s})$ and the late peak $(1.41-1.48 \mu \mathrm{s})$ are shown by the gray areas in the inset in Fig. 2. The solid line is the sum of two Gaussian functions (dotted lines) used to fit the two peaks and identify the time ranges.

Before proceeding to the analysis of the dihedral angle for the two subsets of oriented molecules, we provide an additional argument to corroborate the interpretation that the two-peak structure of the $\mathrm{F}^{+}$signal indeed results from forward- and backward-pointing molecules and not from some artifact in the data acquisition (PImMS camera dead time and microchannel plate voltage ringings). The argument relies on determining correlations between the TOF of different ion species, implemented by a covariance analysis of the TOF spectra [23].

The covariance map is displayed in Fig. 3 and contains a wealth of information not present in the event-averaged TOF spectra. For instance, it is seen that the early $\mathrm{N}^{+}$peak is centered at $1.185 \mu \mathrm{s}$ [Fig. 3(c)], just at the position of the right shoulder of the $\mathrm{C}^{+}$peak. The pertinent observations in the covariance map are that the late $\mathrm{F}^{+}$ions are correlated with the late $\mathrm{N}^{+}$and $\mathrm{N}^{2+}$ ions [Figs. 3(b) and 3(c)] and with the early $\mathrm{Br}^{+}$and $\mathrm{Br}^{2+}$ ions [Figs. 3(d) and 3(e)]. Likewise, early $\mathrm{F}^{+}$ ions are correlated with early $\mathrm{N}^{+}$and $\mathrm{N}^{2+}$ ions and with late $\mathrm{Br}^{+}$and $\mathrm{Br}^{2+}$ ions. These well-resolved correlations, which are hidden in the event-averaged TOF spectra (Fig. 2), show that the recoils of the $\mathrm{F}^{+}, \mathrm{Br}^{+}$, and $\mathrm{N}^{+}$ions reflect the structure

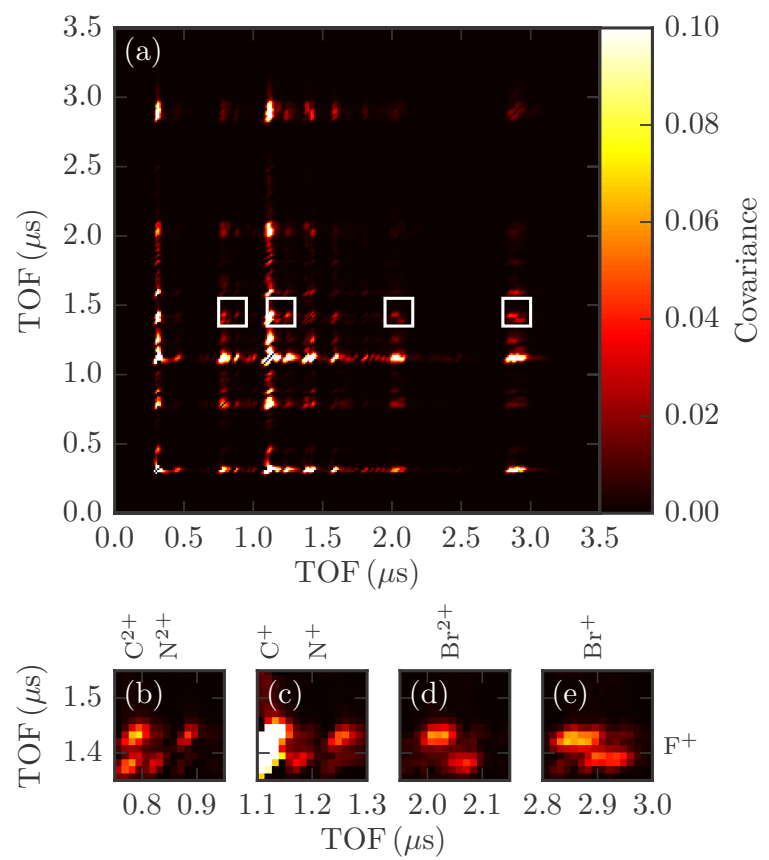

FIG. 3. (Color online) Time-of-flight covariance map of 1D aligned BFCbP. (b)-(e) show magnifications of selected regions (marked by white squares) of the covariance map. In each panel the range of the color scale is adjusted to emphasize the important features.

of the parent molecule depicted in Fig. 1. Therefore, the TOF distribution within the signal belonging to each of these ion species will be indicative of the molecular orientation at the time of the probe pulse.

The remaining part of the data analysis concerns the determination of the dihedral angle for each of the two oriented subsets of molecules. Previously we have shown that for BFCbP molecules (and for very similar molecules) aligned in either one or three dimensions $\phi_{\mathrm{d}}$ can be obtained by measuring the angle between the velocity vectors of $\mathrm{Br}^{+}$and $\mathrm{F}^{+}$ions from the same molecule. In practice this is accomplished by a covariance analysis of the angular distributions of the $\mathrm{F}^{+}$and $\mathrm{Br}^{+}$ions from the 2D images as discussed in Refs. [14,15]. Here the analysis is completed separately for the $\mathrm{F}^{+}$ions in the early peak and in the late peak. Thus, the covariance map of $\theta_{\mathrm{F}^{+}}$and $\theta_{\mathrm{Br}^{+}}$, where $\theta_{\mathrm{F}^{+}}$and $\theta_{\mathrm{Br}^{+}}$are the emission directions of the $\mathrm{F}^{+}$and $\mathrm{Br}^{+}$ions in the detector plane with respect to the upward vertical, is calculated separately for the $\mathrm{F}^{+}$ions in the early peak with all the $\mathrm{Br}^{+}$ions and then for the $\mathrm{F}^{+}$ions in the late peak with all the $\mathrm{Br}^{+}$ions.

The covariance map for the early $\mathrm{F}^{+}$peak is displayed in Fig. 4(a) and it shows pronounced covariances near lines described by

$$
\theta_{\mathrm{F}^{+}}=\theta_{\mathrm{Br}^{+}}+\theta_{\text {const }},
$$

where $\theta_{\text {const }}$ takes four values in the range $40^{\circ}-320^{\circ}$. A similar covariance map is obtained for the late $\mathrm{F}^{+}$peak. To further analyze these correlations, the angular covariance map is integrated along lines of constant $\theta_{\mathrm{F}^{+}}-\theta_{\mathrm{Br}^{+}}$[lines parallel to the diagonal in Fig. 4(a)]. This projection is the sum of the covariances of entries in which $\theta_{\mathrm{F}^{+}}-\theta_{\mathrm{Br}^{+}}$ 

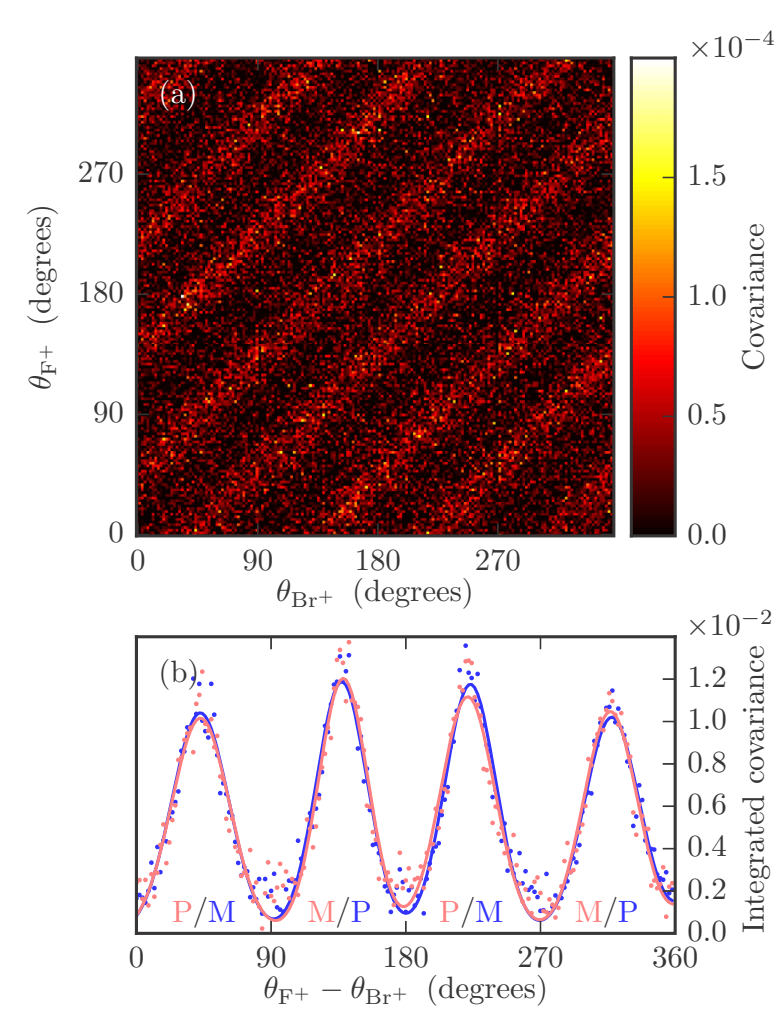

FIG. 4. (Color online) (a) Covariance map of the angular distribution from the $\mathrm{F}^{+}$ions in the early peak and the angular distribution from all the $\mathrm{Br}^{+}$ions. Each broad line of high intensity parallel to the diagonal arises from a single enantiomer. (b) Integration of the covariance maps along lines of constant $\theta_{\mathrm{F}^{+}}-\theta_{\mathrm{Br}^{+}}$with the light red (dark blue) points showing the result for the early (late) $\mathrm{F}^{+}$ion peak. Fits of four Gaussian functions to the experimental data are shown as solid lines with corresponding colors. For both the early peak data and the late peak data each enantiomer gives rise to two peaks in (b). The enantiomers are labeled $P$ or $M$ below the peaks in the same color as the experimental data.

has a certain value. The entries with the highest integrated covariance thus correspond to the most probable difference in the angle detected between the $\mathrm{F}^{+}$and $\mathrm{Br}^{+}$ions. The integrated covariance as a function of $\theta_{\mathrm{F}^{+}}-\theta_{\mathrm{Br}^{+}}$is shown as closed circles in Fig. 4(b) for the early part of the $\mathrm{F}^{+}$ions (light red) and for the late part of the $\mathrm{F}^{+}$ions (dark blue). A sum of four Gaussian functions (solid lines) was fitted to each data set. From the fits the centers of the four peaks are found at approximately $43^{\circ}, 138^{\circ}, 222^{\circ}$, and $317^{\circ}$.

As mentioned, the dihedral angle is the difference between the emission angles of the $\mathrm{F}^{+}$and $\mathrm{Br}^{+}$ions and the centers of the peaks in Fig. 4(b) therefore provide an experimental value of the average dihedral angle $\left\langle\phi_{\mathrm{d}}\right\rangle$. For each molecule undergoing Coulomb explosion an $\mathrm{F}^{+}$ion can be correlated with $\mathrm{a} \mathrm{Br}^{+}$ion from the nearby or the distant $\mathrm{Br}$ site [see Figs. 1(b), 1(c), 1(e), and 1(f)]. This will produce a pair of peaks in Fig. 4(b), one at $\phi_{\mathrm{d}}$ and one at $180^{\circ}+\phi_{\mathrm{d}}$. With this in mind, we stress the central point of the paper, namely, that for the molecules with their $\mathrm{CN}$ end pointing toward the detector (light red data) the peaks at $138^{\circ}$ and at $317^{\circ}$ identifies the $M$ enantiomer and the peaks at $43^{\circ}$ and $222^{\circ}$ the $P$ enantiomer, as labeled in Fig. 4(b). For the molecules with their CN end pointing away from the detector (dark blue data) the situation is reversed, i.e. the peaks at $43^{\circ}$ and $222^{\circ}$ identify the $M$ enantiomer and the peaks at $138^{\circ}$ and $317^{\circ}$ the $P$ enantiomer. With this assignment, the result of the measurement is that $\left\langle\phi_{\mathrm{d}}\right\rangle$ is $42.6 \pm 0.3^{\circ}$ for the $M$ and $42.1 \pm 0.3^{\circ}$ for the $P$ enantiomer. We note that the value of $\left\langle\phi_{\mathrm{d}}\right\rangle$ is larger than the equilibrium dihedral angle calculated. This experimental overestimate is likely due to a slight deviation from the axial recoil in the Coulomb explosion process [14].

The total signal from the $M$ (or $P$ ) enantiomer is given by the sum of the two peaks pertaining to the $M$ (or $P$ ) enantiomer in the covariance plot of Fig. 4(b). For both molecules with the $\mathrm{CN}$ end pointing toward or away from the detector we find that the $M$ and the $P$ enantiomer signals are identical within the experimental uncertainty $( \pm 0.5 \%)$. Two factors contribute to the signal of each enantiomer: their initial population in the molecular beam and any circular dichroism effect in the probe process. The molecular sample used to produce the molecular beam is a racemic mixture and therefore the populations of the two enantiomers are expected to be identical in the experiment. The equal signals measured for the $M$ and the $P$ enantiomers point to no difference in the ionization probabilities for the two enantiomers and thus no circular dichroism effects. Finally, the experiment was repeated with the opposite helicity of the probe pulse and identical results were obtained.

\section{CONCLUSION}

The method described in this paper represents two major advantages compared to other known techniques for determining the AC of chiral molecules. First, it takes less than 15 min to record data that allow a precise determination of the AC despite the repetition rate of the measurements being only $20 \mathrm{~Hz}$. Such an acquisition time is already sufficient to enable time-resolved studies of, for instance, the interconversion of one enantiomer to its mirror form during the natural femtosecond-picosecond duration of the process [24-26]. Recent advances in the repetition rate of laser-induced alignment experiments [27] will reduce the acquisition time, or alternatively increase the time sampling, by a factor 10 or more. Second, the method will apply to a range of molecules even when their size and complexity leads to many different ion species after the Coulomb explosion. Axial chiral molecules of which BFCbP is an example provide a long list of interesting and suitable candidates including other biphenyls, tolane, phenylpyrrole, and various chiral allenes [28]. Furthermore, the method should also work for a number of molecules with a chiral center rather than a chiral axis. In contrast to BFCbP, some of the other molecular species can be synthesized with an enantiomeric excess of either the $M$ or the $P$ configuration. Hereby nonracemic samples are created and our method should be able to determine the enantiomeric excess ratio accurately.

\section{ACKNOWLEDGMENTS}

H.S. acknowledges European Research Council-AdG (Project No. 320459, DropletControl), Carlsbergfondet, and Natur og Univers, Det Frie Forskningsråd. M.B. acknowledges support from the Engineering and Physical Sciences Research 
Council via Programme Grant No. EP/L005913/1, the EU through grant FP7 ITN Seventh Framework Programme, ICONIC (Project Grant No. 238671), STFC through a Science and Technology Facilities Council, PNPAS award and a
mini-IPS grant (Grant No. ST/J002895/1), and a proof of concept grant from ISIS Innovation Ltd. A.L. thanks the Deutsche Forschungsgemeinschaft for support via Grant No. La 3209/1-1.
[1] J. M. Bijvoet, A. F. Peerdeman, and A. J. van Bommel, Nature (London) 168, 271 (1951).

[2] P. J. Stephens, F. J. Devlin, and J.-J. Pan, Chirality 20, 643 (2008).

[3] L. D. Barron, L. Hecht, I. H. McColl, and E. W. Blanch, Mol. Phys. 102, 731 (2004).

[4] N. Böwering, T. Lischke, B. Schmidtke, N. Müller, T. Khalil, and U. Heinzmann, Phys. Rev. Lett. 86, 1187 (2001).

[5] G. A. Garcia, L. Nahon, M. Lebech, J.-C. Houver, D. Dowek, and I. Powis, J. Chem. Phys. 119, 8781 (2003).

[6] C. Lux, M. Wollenhaupt, T. Bolze, Q. Liang, J. Köhler, C. Sarpe, and T. Baumert, Angew. Chem. Int. Ed. 51, 5001 (2012).

[7] C. S. Lehmann, N. B. Ram, I. Powis, and M. H. M. Janssen, J. Chem. Phys. 139, 234307 (2013).

[8] D. Patterson, M. Schnell, and J. M. Doyle, Nature (London) 497, 475 (2013).

[9] V. A. Shubert, D. Schmitz, D. Patterson, J. M. Doyle, and M. Schnell, Angew. Chem. Int. Ed. 53, 1152 (2014).

[10] T. Kitamura, T. Nishide, H. Shiromaru, Y. Achiba, and N. Kobayashi, J. Chem. Phys. 115, 5 (2001).

[11] P. Herwig, K. Zawatzky, M. Grieser, O. Heber, B. Jordon-Thaden, C. Krantz, O. Novotný, R. Repnow, V. Schurig, D. Schwalm, Z. Vager, A. Wolf, O. Trapp, and H. Kreckel, Science 342, 1084 (2013).

[12] M. Pitzer, M. Kunitski, A. S. Johnson, T. Jahnke, H. Sann, F. Sturm, L. P. H. Schmidt, H. Schmidt-Böcking, R. Dörner, J. Stohner, J. Kiedrowski, M. Reggelin, S. Marquardt, A. Schießer, R. Berger, and M. S. Schöffler, Science 341, 1096 (2013).

[13] A. D. McNaught and A. Wilkinson, IUPAC. Compendium of Chemical Terminology, 2nd ed. (Blackwell Scientific, Oxford, 1997).

[14] C. S. Slater, S. Blake, M. Brouard, A. Lauer, C. Vallance, J. J. John, R. Turchetta, A. Nomerotski, L. Christensen, J. H. Nielsen, M. P. Johansson, and H. Stapelfeldt, Phys. Rev. A 89, 011401 (2014).

[15] L. Christensen, J. H. Nielsen, C. B. Brandt, C. B. Madsen, L. B. Madsen, C. S. Slater, A. Lauer, M. Brouard, M. P. Johansson, B. Shepperson, and H. Stapelfeldt, Phys. Rev. Lett. 113, 073005 (2014).
[16] F. Filsinger, J. Küpper, G. Meijer, L. Holmegaard, J. H. Nielsen, I. Nevo, J. L. Hansen, and H. Stapelfeldt, J. Chem. Phys. 131, 064309 (2009).

[17] J. L. Hansen, J. J. Omiste, J. H. Nielsen, D. Pentlehner, J. Küpper, R. González-Férez, and H. Stapelfeldt, J. Chem. Phys. 139, 234313 (2013).

[18] J. L. Hansen, J. H. Nielsen, C. B. Madsen, A. T. Lindhardt, M. P. Johansson, T. Skrydstrup, L. B. Madsen, and H. Stapelfeldt, J. Chem. Phys. 136, 204310 (2012).

[19] A. T. J. B. Eppink and D. H. Parker, Rev. Sci. Instrum. 68, 3477 (1997).

[20] A. Nomerotski, M. Brouard, E. Campbell, A. Clark, J. Crooks, J. Fopma, J. J. John, A. J. Johnsen, C. Slater, R. Turchetta, C. Vallance, E. Wilman, and W. H. Yuen, J. Instrum. 5, C07007 (2010).

[21] J. J. John, M. Brouard, A. Clark, J. Crooks, E. Halford, L. Hill, J. W. L. Lee, A. Nomerotski, R. Pisarczyk, I. Sedgwick, C. S. Slater, R. Turchetta, C. Vallance, E. Wilman, B. Winter, and W. H. Yuen, J. Instrum. 7, C08001 (2012).

[22] C. Vallance, M. Brouard, A. Lauer, C. S. Slater, E. Halford, B. Winter, S. J. King, J. W. L. Lee, D. E. Pooley, I. Sedgwick, R. Turchetta, A. Nomerotski, J. J. John, and L. Hill, Phys. Chem. Chem. Phys. 16, 383 (2014).

[23] L. J. Frasinski, K. Codling, and P. A. Hatherly, Science 246, 1029 (1989).

[24] C. B. Madsen, L. B. Madsen, S. S. Viftrup, M. P. Johansson, T. B. Poulsen, L. Holmegaard, V. Kumarappan, K. A. Jørgensen, and H. Stapelfeldt, Phys. Rev. Lett. 102, 073007 (2009).

[25] C. B. Madsen, L. B. Madsen, S. S. Viftrup, M. P. Johansson, T. B. Poulsen, L. Holmegaard, V. Kumarappan, K. A. Jørgensen, and H. Stapelfeldt, J. Chem. Phys. 130, 234310 (2009).

[26] D. Kröner and B. Klaumünzer, Chem. Phys. 338, 268 (2007).

[27] S. Trippel, T. G. Mullins, N. L. Müller, J. S. Kienitz, K. Długołcki, and J. Küpper, Mol. Phys. 111, 1738 (2013).

[28] E. L. Eliel and A. H. Wilen, Stereochemistry of Organic Compounds (Wiley, New York, 1994). 